\title{
Prognostic Factors, Treatment and Outcome in a Turkish Population with Endometrial Stromal Sarcoma
}

\author{
Ayla Donertas ${ }^{1}$, Umit Nayki $^{2 *}$, Cenk Nayki ${ }^{2}$, Pasa Ulug ${ }^{2}$, Emre Gultekin ${ }^{1}$, Yusuf \\ Yildirim $^{1,2}$
}

\begin{abstract}
Purpose: To analyze treatment modalities and prognostic factors in patients with Stage I-II endometrial stromal sarcoma (ESS). Materials and Methods: Twenty four patients (nineteen with low-grade ESS [LGESS] and five with high-grade ESS [HGESS]) were assessed retrospectively in terms of general characteristics, prognostic factors, treatment methods and survival. Results: Twenty patients were at Stage I and three were at Stage II. The stage of one patient could not be determined. With respect to age and comorbidity, no statistically significant difference was found among disease-free survival (DFS) $(p=0.990 ; p=0.995)$. However, DFS was significantly shorter in Stage II than Stage I patients $(p=0.002)$. It was also significantly shorter in HGESS patients than in LGESS patients $(p=0.000$ ). There was no statistically significant differences among the overall survival (OVS) times of patients with respect to age at diagnosis and comorbid disease $(p=0.905 ; p=0.979)$ but OVS was significantly shorter in patients with HGESS $(p=0.00)$ and Stage II disease ( $p=0.001)$. No statistically significant difference was found with respect to OVS between patients who received radiotherapy (RT) and those who did not receive $R T$ ( $p=0.055$ ). It was not statistically possible to include other treatment modalities in the analysis because of the small sample size. Conclusions: Grade and stage of a tumour were found to be the most important prognostic factors. It was not possible to determine the optimal surgical method and the effect of adjuvant treatment since the number of cases was insufficient.
\end{abstract}

Keywords: Endometrial stromal sarcoma - prognostic factors - treatment methods

Asian Pac J Cancer Prev, 16 (3), 881-887

\section{Introduction}

Endometrial stromal sarcoma (ESS) is a very rare cancer that develops from endometrial connective tissue and is seen in $0.2-1 \%$ of all uterine malignancies and in $10-15 \%$ of all uterine sarcomas (Durnalı et al., 2012; Ghaemmaghami et al., 2008, Larson et al., 1990; Livi et al., 2004). ESS was first classified in 1966 by Norris and Taylor as low-grade (well/moderately differentiated) ESS [LGESS] and high-grade (poorly differentiated) ESS [HGESS] according to its pathological characteristics. However, since anaplastic tumour cells never show any stromal differentiation and exhibit a different clinical behaviour in high-grade ESS, the World Health Organization (WHO) published a new tumour classification in 2003. According to this classification, ESSs were divided into three groups: Benign stromal nodule, ESS (LGESS in the former classification) and undifferentiated endometrial sarcoma (UES) (HGESS in the former classification) (Tavassoli et al., 2003; Moinfar et al., 2007; Nicholas et al., 2007). The differentiation between ESS and UES is based on the number of mitoses, nuclear pleomorphism, atypia, and necrosis (Moinfar et al., 2007; Gadducci et al., 2008).

ESSs are low-grade tumours resembling endometrial stroma cells at the proliferative phase and they show no apparent nuclear atypia ( Brooks et al., 2004). They stem from the stroma of the endometrium and mostly (90\%) infiltrate the myometrium, and lymphatic extension is common. UESs, on the other hand, are aggressive malignant tumours and have noticeable cytological atypia and high mitotic activity. They do not exhibit endometrial stromal differentiation (Moinfar et al., 2007; Gadducci et al., 2008). The treatment and prognosis of ESSs vary according to tumour type. In this study, we aimed at investigating the clinicopathological characteristics, treatment methods, and prognostic factors in ESSs.

\section{Materials and Methods}

Twenty- four patients who had previously been diagnosed with ESS at the Gynaecology and Obstetrics Clinic of Izmir Tepecik Training and Research Hospital between 1992 and 2010 were assessed retrospectively 
in terms of general characteristics, prognostic factors, treatment methods and survival. This study was approved by the ethics committee of the hospital. Patient information was recorded with respect to age, menopause, initial complaint, comorbidity, type and date of surgery, grade, number of mitoses (NM), stage, nuclear atypia, lymphovascular area invasion, presence of necrosis, adjuvant treatment methods performed, location and date of relapse, date of final clinical examination, disease-free survival (DFS) time and overall survival (OVS) time.

For histopathological classification, the patients were divided into two groups: Those with low-grade ESS (LESS) and those with high grade ESS (HESS). Since the term UES was defined in WHO's 2003 classification, Norris and Taylor's original term HGESS was used instead of the term UES. The patients were also divided into three groups with respect to NM in 10 High Power Fields (HPF) $(\mathrm{NM} \leq 10 / 10 \mathrm{HPF}, \mathrm{NM}>10 / 10 \mathrm{HPF}$, and NM not mentioned). The presence of necrosis and lymphovascular area invasion was noted as "yes/no/not mentioned".

The 2009 FIGO (International Federation of Gynecology and Obstetrics) staging system revised for ESS and leiomyosarcoma (LMS) was used for staging (Prat.,2009).

The primary treatment for all patients included in the study was surgery and the operations were divided into three groups: Total Abdominal Hysterectomy (TAH), TAH+Bilateral Salpingo-oophorectomy ( $\mathrm{BSO}) \pm \mathrm{Omentectomy}$ and $\mathrm{TAH}+\mathrm{BSO}+\mathrm{Omentectomy+Pelvic-Paraaortic}$ Lymphadenectomy (PPLA). The patients were also divided into four groups with respect to their postoperative adjuvant treatments: Group1. Adjuvant radiotherapy (RT) (external \pm intracavitary), Group 2. Adjuvant RT+chemotherapy (CT), Group 3. Adjuvant CT and Group 4. Follow-up alone (adjuvant therapy deemed unnecessary).

Relapses were assessed as local, distant or local+distant relapses. The therapies used during relapses were classified as RT, CT, hormonal therapy (HT) or a combination of various treatment methods.

All patients were monitored with quarterly checks for 2 years after the diagnosis, with 4-monthly checks for the following 2 years, with semiannual checks for the following 2 years and with annual checks thereafter. During each check, patients underwent gynaecological examination and pelvic+abdominal ultrasonography. All patients were routinely performed imaging and smear once a year.

OVS time was calculated based on the time elapsed since the surgical operation to the date of the final check. DFS time was based on the time elapsed since the surgical operation to the date of relapse and, for patients who had no relapse, to the date of the final check.

The SPSS (Statistical Package for Social Sciences Chicago, IL, USA) for Windows 20.0 program was used for statistical analyses. Average, standard deviation, ratio and frequency values were calculated. The Kaplan-Meier survival analysis was used for survival analyses and the Log Rank test for their comparison. The Cox Regression test was used for multiple assessments. The results were evaluated within a $95 \%$ confidence interval with significance at $\mathrm{p}<0.05$.

\section{Results}

There were nineteen (79\%) LGESS cases and five (21\%) HGESS cases. The mean age of patients at

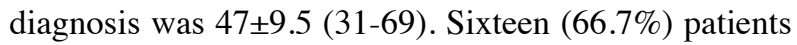
were in the pre/perimenopausal period and eight (28.3\%) in the postmenopausal period. The most frequent initial complaint was irregular vaginal bleeding. Uterine leiomyoma was found in eight patients (four of them had no preoperative endometrial sample, one patient had simple atypia-free endometrial hyperplasia and three patients had benign endometrial samples). There was a comorbid disease accompanying the condition in five patients (one patient had surgery for a brain tumour). Twenty patients $(83.3 \%)$ were assessed as stage I (Fifteen of them were stage IA and five patients were stage IB) and three patients were stage II (all of them were stage IIB). The stage of one patient could not be determined as the pathology report was not available. There were no stage III or IV patients in our study population.

There was no mention of NM in 11 patients and NM
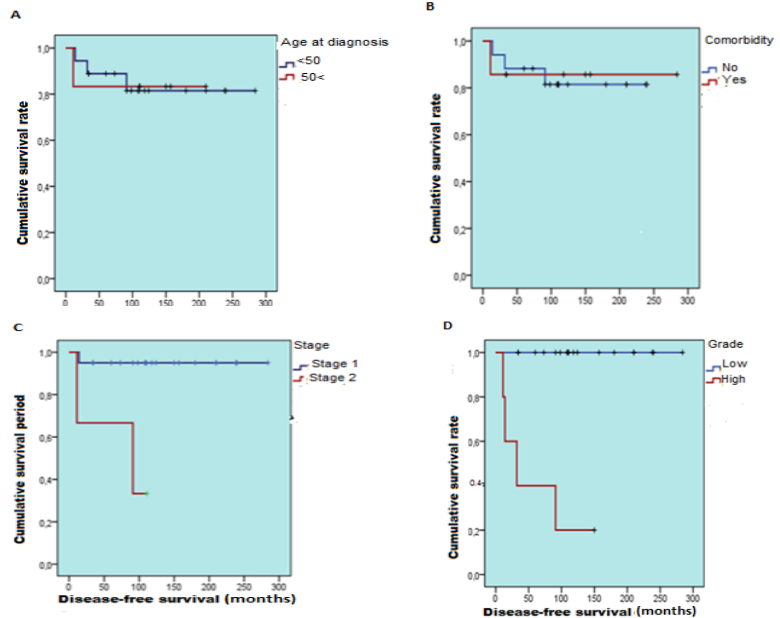

Figure 1. Disease-free Survival According. A) age at diagnosis, B) comorbidity, C) stage and $\mathbf{D})$ grade
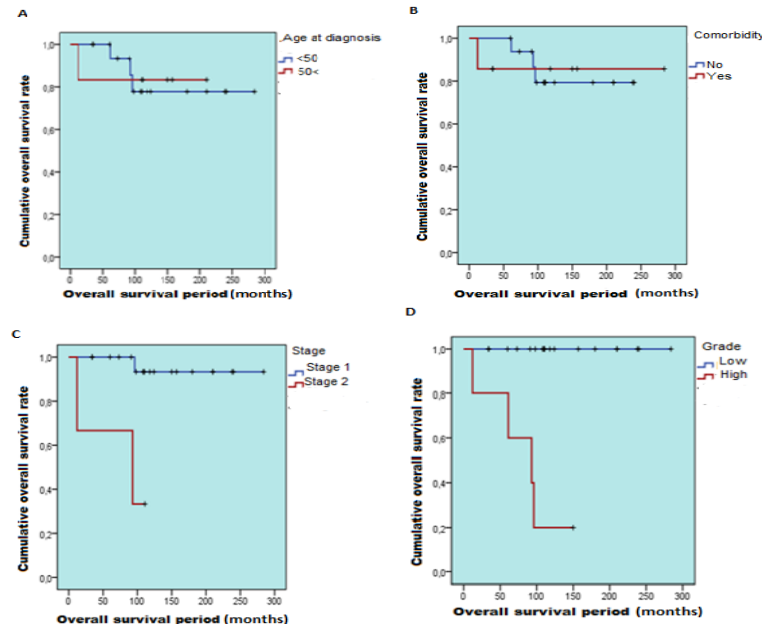

Figure 2. Cumulative Survival According. A) age at diagnosis, B) comorbidity, C) stage and D) grade 
was 50 in one patient. NM was between 0 and 5 in the other 12 patients. NM was not included in the statistical evaluation as a prognostic factor, because there was only one case with "NM>10". Moreover, since there were insufficient records regarding the presence of necrosis or lymphovascular area invasion, statistical analyses could not be carried out for these parameters contrary to initial plans.

Only TAH was administered to three patients (12.5\%), TAH+BSO+Omentectomy to 18 patients $(75.0 \%)$ and TAH+BSO+Omentectomy+PPLA to 3 patients (12.5\%).

Table 1. Clinico-Pathological Characteristics of the Patients

\begin{tabular}{|c|c|c|}
\hline Mean \pm sd & $\mathrm{n}$ & $\%$ \\
\hline Age at diagnosis & & \\
\hline Comorbidity & 4 & 16.70 \\
\hline \multicolumn{3}{|l|}{ Menopausal status } \\
\hline Pre/Postmenopause & 16 & 66.70 \\
\hline Postmenopause & 8 & 33.30 \\
\hline \multicolumn{3}{|l|}{ Symptoms } \\
\hline Abnormal bleeding & 9 & 37.50 \\
\hline Symptoms of compression & 5 & 20.80 \\
\hline Postmenopausal bleeding & 4 & 16.70 \\
\hline Pelvic pain & 3 & 12.50 \\
\hline Asymptomatic & 3 & 12.50 \\
\hline \multicolumn{3}{|l|}{ Preoperative sampling } \\
\hline None & 7 & 29.20 \\
\hline Bening & 10 & 41.70 \\
\hline Malignant & 7 & 29.20 \\
\hline \multicolumn{3}{|l|}{ Grade } \\
\hline Low & 19 & 79.20 \\
\hline High & 5 & 20.80 \\
\hline \multicolumn{3}{|l|}{ Stage } \\
\hline Stage 1 & 20 & 83.30 \\
\hline Stage 2 & 5 & 12.50 \\
\hline \multicolumn{3}{|l|}{ Operation } \\
\hline TAH & 3 & 12.50 \\
\hline TAH+BSO+OMENTECTOMY & 18 & 75.00 \\
\hline TAH+BSO+OMENTECTOMY+PPLA & 3 & 12.50 \\
\hline \multicolumn{3}{|l|}{ Adjuvant therapy } \\
\hline ERT+IRT & 10 & 41.70 \\
\hline $\mathrm{ERT}+\mathrm{CT}$ & 1 & 4.20 \\
\hline $\mathrm{CT}$ & 1 & 4.20 \\
\hline None & 12 & 50.00 \\
\hline
\end{tabular}

*TAH: Total abdominal hysterectomy ERT: External radiotherapy sd: Standard deviation; BSO: Bilateral salphingo-oophorectomy IRT: Internal radiotherapy; PPLA: Pelvic-para-aortic lymph adenectomy CT: Chemotherapy
No omentum involvement was found in any of the six patients who were administered omentectomy, whereas lymph samples were found to have a benign character in three patients who were administered lymphadenectomy. Only RT was given to ten patients (41.7\%) (external to eight patients and external+intracavitary to two patients), three cycles of the ifosfamide+mesna+adriamycin (IMA) chemotherapy protocol following RT to one patient (4.2\%), six cycles of postoperative IMA chemotherapy protocol without RT to one patient (4.2\%) and 12 patients (50\%) were not given any adjuvant treatment and were monitored as they were. No adjuvant HT was given to any of the patients, but a single patient (the patient with HGESS who developed lung metastasis) was given Megesterol acetate 2x160 mg daily for 3 months for relapse treatment.

The median follow-up period was 110 months (12 months-248 months). During follow-up, relapses were seen in four patients $(16.7 \%)$, local relapses (pelvic relapse lump and peritonitis carcinomatosis) in two patients and distant organ relapses (lung and bone) in the other two. All of these patients had HGESS. All four patients who developed relapses died due to the disease. At the time of writing, the other 20 patients (including one patient who was diagnosed with HGESS) are alive without the disease. The demographic and clinico-pathological characteristics of the patients are given in Table 1 .

The clinical characteristics of those patients who developed relapses and died were examined separately (Table 2). The first case: The patient was diagnosed with stage IB HGESS at the age of 44, was administered standard curative surgery (TAH+BSO) and then was given external RT. She developed isolated lung metastasis at Month 14 and was treated with six cycles of Doxorubicin+Cisplatin chemotherapy as a curative treatment. After 74 months, a miliary lung metastasis was seen for the second time and she was given Megestrol acetate $160 \mathrm{mg}$ twice daily for 3 months; when sufficient response could not be achieved, three cycles of the Paclitaxel+Cisplatin chemotherapy protocol were administered. The patient died at month 96 .

The second case: The patient was diagnosed with stage IIB HGESS at the age of 48; she underwent optimal surgery (TAH+BSO+Omentectomy+PPLA) followed by combined externaland internal $\mathrm{RT}+$ three cycles of IMA chemotherapy. The patient developed peritonitis

Table 2. Clinico-Pathological Characteristics of Patients Who Died

\begin{tabular}{lcccc}
\hline & EX 1 & EX 2 & EX 3 & EX 3 \\
\hline Age at diagnosis & 44 & 48 & 41 & 55 \\
Grade & High & High & High & High \\
Stage & IB & IIB & IIB \\
Operation & TAH+BSO & TAH+BSO+PPLA & TAH & TAH+BSO \\
Adjuvant therapy & ERT & ERT+IRT+3 Cure CT & ERT+IRT & ERT* \\
Relapse & Lung Met 2 times & P. carcinomatosis & Bone Met & Pelvic mass \\
Therapy at relapse & 6 cure CT & - & & - \\
-First relapse & 3 months Megace & CT planned,but & ERT+IRT & CT planned, but \\
-Second relapse & "followed by 3cure CT" & not administered & & not administered \\
Disease free survival & 14 months & 91 months & 32 months & 11 months \\
Total survival & 96 months & 93 months & 61 months & 12 months \\
\hline
\end{tabular}

*Chemotherapy was planned, but could not be administered because of the general status of the disorder; TAH: Total abdominal hysterectomy BSO: Bilateral salphingooophorectomy; ERT: External radiotherapy IRT: Internal radiotherapy CT: Chemotherapy 
carcinomatosis at Month 91 and died at Month 93.

The third case: The patient was diagnosed with HGESS at an unknown stage at the age of 41 and was administered TAH followed by RT. Widespread bone metastases developed in the patient at Month 32 and palliative RT and CT were administered. The patient died at Month 61 .

The fourth case: The patient was diagnosed with stage IIB HGESS at the age of 55 and had comorbid diseases. The patient was administered $\mathrm{TAH}+\mathrm{BSO}+\mathrm{Omentectomy}$ followed by external RT and an IMA chemotherapy protocol was planned. The planned chemotherapy was aborted due to impaired general condition associated with comorbidity. A pelvic relapse lump developed in the patient at Month 11 and she died at Month 12.

DFS periods were between 11 and 284 months with an average of 142 months. The first of the two local relapses developed within the first year and the other in the eighth year. Both of the two distant metastases developed in the first 3 years. At the end of the study, the 2-year DFS rate was found to be $87 \%$, the 3-year DFS rate $87 \%$, the 5-year DFS rate $79 \%$ and the 10 -year DFS rate $82 \%$.

No statistically significant difference was found between the DFS times of patients in terms of age $(\leq 50$ $v s>50)($ Log-rank 0.0, $\mathrm{p}=0.990>0.05)($ Graph 1A). No statistically significant difference was found between the DFS times of patients with comorbid diseases (four patients) and those without them (20 patients) (Log-rank $0.0, \mathrm{p}=0.945>0.05)$ (Graph 1B). The DFS was significantly lower in Stage 2 patients than in Stage 1 patients (Logrank 9.2, $\mathrm{p}=0.002<0.01)(\mathrm{Graph} 1 \mathrm{C})$. The DFS was significantly lower in HGESS patients than in LGESS patients in statistical terms (Log-rank 21.3, $\mathrm{p}=0.000<0.01$ ) (Graph 1D).

OVS times were between 12 and 284 months; no median survival time was calculated given that 21 patients were still alive. The mean OVS time was 241.68 months (standard error 19.11). One patient died in the fifth year of follow-up period. The 2-year OVS rate was found to be $96.3 \%$, the 3 -year OVS rate $96 \%$, the 5-year OVS rate $72 \%$ and the 10-year OVS rate $80 \%$.

No statistically significant difference was found between the OVS times of patients aged $\leq 50$ and $>50$ at diagnosis (Log-rank 0.0, p=0.905>0.05) (Graph 2A). No statistically significant difference was found between the OVS times of patients with comorbid diseases and those without them (Log-rank 0.0, p=0.979>0.05) (Graph 2B). OVS was significantly shorter in high-grade patients than in low-grade patients (Log-rank 18.7, $\mathrm{p}=0.00<0.01$ ) (Graph 2C). It was significantly shorter in Stage 2 patients than in Stage 1 patients (Log-rank 10.3, p=0.001<0.01) (Graph 2D).

No statistically significant difference was found between patients who received RT and those who did not in terms of OVS time (Log-rank 4.8, $\mathrm{p}=0.055>0.05)$. It was not statistically possible to include the other treatment modalities in the analysis because of the small number of cases (a single patient receiving CT only and another receiving $\mathrm{RT}+\mathrm{CT}$ in combination). The OVS time was significantly shorter in patients with relapses than in patients with no relapse (Log-rank 28.6, $\mathrm{p}=0.000<0.001$ ).

\section{Discussion}

ESSs are rarely seen mesenchymal tumours and constitute $0.2-1 \%$ of all uterine malignancies Koss et al., 1965; Echt et al., 1990; Larson et al., 1990). They usually affect women between 40 and 55 years of age, who are in the pre/perimenopause period (Xue and Cheung, 2011). In the patient group we examined, the youngest patient was 31 years old and the oldest 69 years old, with a mean age of 47 at diagnosis. A total of $66.7 \%$ of these patients were in premenopause and $28.3 \%$ of them in postmenopause and these results are similar to those in the literature (Xue and Cheung, 2011). While age has been considered a prognostic factor in some publications (Chan et al., 2008), no relationship was found between age and prognosis in other publications (Nordal et al., 1996). Likewise we found no relationship between age and survival in our study.

ESS has no specific clinical symptoms. Patients often present with the complaint of vaginal bleeding. In our study, the patients' most frequent initial complaints were vaginal bleeding (54.2\%) and compression symptoms $(20.8 \%)$. Twelve percent of the patients were asymptomatic. This clinical presentation is similar to the figures noted by Haberal et al. (2003).and Li et al. (2008). Sagage et al. (2004) reported that $75 \%$ of patients diagnosed with ESS were initially diagnosed with uterine leiomyoma mistakenly in the preoperative period. We found a lower rate (33\% of cases) in our study. Our study revealed that the rate at which endometrial sampling detected the tumour was $41.2 \%$ (in $7 / 17$ cases). This is close to the rate found by $\mathrm{Li}$ et al. (2008), which was $45.5 \%$.

The rate of extrauterine diseases at the time of diagnosis lies between $15 \%$ and $61 \%$ in the literature (Sagage et al., 2004; Leath et al., 2007). We did not encounter any extrauterine disease at the time of diagnosis in our study.

The effect of systemic diseases on survival and prognosis has been assessed in a few studies and no significant difference has been found (Haberal et al., 2003). Our study also found no significant difference between disease-free and total survival times of patients with comorbid diseases and those with no comorbid diseases.

The grade of a tumour has appeared to be a strong independent prognostic factor in various studies (Ghaemmaghami et al., 2008; Durnali et al., 2012). It is known that HGESSs exhibit more aggressive biologicalclinical behaviour than LGESSs and they result in shorter survival times ( Rauh-Hain et al., 2013). Leath et al. (2007) reported in their multicentre study that LGESS and HGESS are two different clinical entities and should be treated accordingly. The median OVS time was calculated as 80 months for LGESS and 53 months for HGESS. Gadducci et al. (1996) also concluded that the DFS time was significantly longer in patients with LGESS than in patients with HGESS. The DFS and OVS times were also significantly shorter in high-grade patients than in lowgrade patients in our study ( $\mathrm{p}<0.001$ in both). 
Another independent prognostic factor indentified in many studies is tumour stage (Bodner et al., 2001; Geller et al., 2004; Barney et al., 2009). In their study including 310 patients with endometrial stromal tumours, Garg et al. ( 2010) found that the 5-year survival was better in stage IA patients $(100 \%)$ than in stage IB patients (95.3\%).Our study also showed that the DFS and OVS were significantly shorter in stage II patients than in stage 1 patients ( $\mathrm{p}=0.002$ and $\mathrm{p}=0.001$, respectively).

LGESSs are lazy tumours and tend to relapse in late periods. Relapses are most likely in the pelvis and abdomen (Chang et al., 1990; Bodner et al., 2001; Malouf et al., 2010). HGESSs (UESs), on the other hand, are aggressive tumours and may have early relapses (Yamawaki et al., 1997). The rate of recurrence was $17 \%$ for stage I and II ESSs in our study (lower than previously published data). All of the patients in whom relapses were seen had the HGESS histopathology. The rate of developing relapses in the first 3 years was $60 \%$ in HGESS patients.

The standard treatment for ESS is surgery (Goff et al., 1993). TAH+BSO is a standard curative procedure for early stage (stages I and II) and low-grade patients. The role of lymphadenectomy remains controversial ( Reich et al., 2005; Riopel et al., 2005). The rate of lymph node involvement in ESS lies between $10 \%$ and $45 \%$ ( Reich et al., 2005; Leath et al., 2007). The status of lymph nodes is important both for the prognosis and the need for adjuvant treatment. In their retrospective study of 105 patients, Leath et al. (2007) reported that the DFS and OVS times were associated with the residual tumour size in UES and complete surgery (pelvic-para-aortic lymphadenectomy) extended the survival time. However, Barney et al. (2009) investigated 1010 cases retrospectively in their multicentre study and concluded that lymphadenectomy added to primary surgery did not change survival in either grade (low or high). Tanz et al. (2012) reported that lymph node dissection did not provide any extra benefit. Chan et al. (2008), on the other hand, found node positivity in $10 \%$ of the patients who were administered lymphadenectomy. The 5-year survival was markedly shorter in these patients compared to those who did not have any nodal involvement (35.3\% and $80.1 \%$, respectively). In our study, only three patients (two with LGESS and one with HGESS that were known to be malignant preoperatively) were administered lymphadenectomy and no lymph node involvement occurred. There are no studies in the literature regarding omentum involvement and omentectomy except one in which involvement was seen in a single patient (with UES) (Li et al., 2008). In our study, six out of 24 patients were administered a total omentectomy and no involvement occurred. Opinions on ovary-protecting surgery are also contradictory. Chan et al. (2008) demonstrated that protection of ovaries did not affect survival negatively in stage I or stage II ESS patients under 50 years of age (96.2\% vs $91.9 \%$ ) and considered ovary-protecting surgery as an option in young and early stage patients. Amant et al. (2007) reported that the rate of relapse was $25 \%$ (3/12) in early stage women who underwent an oophorectomy and $17 \%(1 / 6)$ in those who did not. Similarly, Li et al. (2005) concluded that BSO did not have any significant impact on DFS or OVS. Chu et al. (2003) and Gadducci et al.
(2008) published similar results. In our study, the ovaries of three patients were protected and a distant metastasis developed in one patient (a high-grade case with unknown stage) in the third year. The other two patients (stage IB low-grade cases) had no relapse and are alive at the time of writing. We suggest that the decision to administer a BSO or not in addition to a hysterectomy in stage I patients at a young age should be made for each individual patient after evaluating the benefit-risk status of the decision. Not allowing the continuation of ovarian activity may be a reasonable approach for these hormone-sensitive tumours.

There are different views in the literature about the need for adjuvant treatment in ESS. There are publications reporting that adjuvant RT has no effect on survival (Bodner et al., 2001; Garg et al., 2010) and also publications saying that it prolongs DFS (Weitmann et al., 2001; Tanz et al., 2012). Li et al. (2008) reported that the rate of local control was $93.8 \%$ in patients who received adjuvant $\mathrm{RT}$ and $57.1 \%$ in those who did not receive adjuvant $\mathrm{RT}$, but stated that $\mathrm{RT}$ did not prolong total survival. Gadducci et al. (2008) also reported that postoperative RT reduced the frequency of local recurrence (but was ineffective in distant metastases), but, had no effect on OVS in any grade. Mansi et al. (1990) suggested administering RT postoperatively in HGESS and leaving it until a relapse treatment in LGESS. In our study, there was no statistically significant difference between patients receiving $\mathrm{RT}$ and those not receiving RT in terms of DFS and OVS times $(p=0.061$ and $p=0.055$, respectively).

The role of adjuvant CT is also unclear. The reason for this is the small patient groups and the fact that one or a few patients in these groups were given chemotherapeutic agents. The first of the two patients who received CT (stage IB, low-grade) in our study received six cycles of CT and has been disease-free for 20 years. The other patient who received adjuvant $\mathrm{RT}$ and $\mathrm{CT}$ in combination was stage IIB high-grade and developed peritonitis carcinomatosis at Month 91 and died 2 months later. In a study conducted by $\mathrm{Li}$ et al. (2008), the chemotherapy protocols with Ifosfamide+Adriamycin+Cisplatin (IAP) or with Vin cristine+Adriamycin+Dacarbazine (VAD) were found to be effective in UESs and reduced the rates of local recurrence. However, when the OVS times were reviewed, no statistically significant difference was found between the groups receiving and not receiving CT. The conclusion derived from that study was that adjuvant chemotherapy should be given to patients who have HGESS and deep myometrial invasion and CT should also be administered to those who develop relapses after resection of the relapsed lump if operable and for the treatment of the relapse if inoperable.

Contrary to HGESSs, LGESSs are hormone-sensitive tumours that express large amounts of oestrogen and progesterone receptors (Berchuck et al., 1990; Gadducci et al., 2011). Although there are publications in the literature about the use of progestin-containing agents and anti-estrogenic agents in advanced stage (stage III and IV) or recurrent ESS patients with positive oestrogen and progesterone receptors, both as adjuvant treatment and relapse treatment (Maluf et al., 2001; Spano et al., 
2003), there is a need for further prospective studies on appropriate hormonal therapy modalities and duration of treatments. Hormonal therapy has no place in HGESSs (since they are ER and PR negative in general) (Ihnen et al., 2007; Casali et al., 2010). We did not use HT in any patient as an adjuvant treatment except in one patient with relapse, to whom we administered Megestrol acetate for 3 months, but could not achieve a good response.

In conclusion, ESSs are rarely seen tumors and data on these is limited in the literature. The studies on this issue are retrospective studies performed on a few patient groups. For this reason, no consensus could be achieved about early diagnosis, prognostic factors, tumour behaviour, or treatment management modality. Our results show that tumor grade and stage is the most important prognostic factor. We found no effect of menopause or age on survival. Since the number of patients was inadequate, it was not possible in our study to determine the optimal surgery type or to decide whether or not ovaries should be protected or lymphadenectomy or omentectomy included in the operation. No management modality has been specified in the literature due to similar restrictions. The benefit of adjuvant treatment is unclear. In view of this, there is a need for larger scale prospective studies on endometrial stromal tumours.

\section{References}

Amant F, De Knijf A, Van Calster B, et al (2007). Clinical study investigating the role of lymphadenectomy, surgical castration and adjuvant hormonal treatment in endometrial stromal sarcoma. Br J Cancer, 97, 1194-9.

Barney B, Tward JD, Skidmore T, Gaffney DK (2009). Does radiotherapy or lymphadenectomy improve survival in endometrial stromal sarcoma? Int J Gynecol Cancer, 19, 1232-8.

Berchuck A, Rubin SC, Hoskins WJ, et al (1990). Treatment of endometrial stromal tumors. Gynecol Oncol, 36, 60-5.

Bodner K, Adler BB, Obermair A, et al (2001). Prognostic parameters in endometrial stromal sarcoma: A clinicopathologic study in 31 patients. Gynecol Oncol, 81, 160-5.

Brooks SE, Zhan M, Cote T, Baquet CR(2004). Surveillance, epidemiology, and end results analysis of 2677 cases of uterine sarcoma 1989-1999. Gynecol Oncol, 93, 204.

Casali PG, Blay JY (2010). Soft tissue sarcomas: ESMO clinical practice guidelines for diagnosis, treatment and follow-up. Ann Oncol, 21, 198-203.

Chan JK, Kawar NM, Shin JY, et al (2008). Endometrial stromal sarcoma: a population- based analysis. Br J Cancer, 99, 1210-5.

Chang KL, Crabtree GS, Lim-Tan SK, Kempson RL, Hendrickson MR (1990). Primary uterine endometrial stromal neoplasms. A clinicopathologic study of 117 cases. Am J Surg Pathol, 14, 415-38.

Chu MC, Mor G, Lim C, et al (2003). Low-grade endometrial stromal sarcoma: hormonal aspects. Gynecol Oncol, 90, 170-6.

Durnalı A, Tokluoğlu S, Özdemir N, et al (2012). Prognostic factors and treatment outcomes in 93 patients with uterine sarcoma from 4 centers in Turkey. Asian Pac J Cancer Prev, 13, 1935-41

Echt G, Jepson J, Steel J, et al (1990). Treatment of uterine sarcomas. Cancer, 66, 35-9.
Gadducci A, Sartori E, Landoni F,et al (1996). Endometrial stromal sarcoma: analysis of treatment failures and survival. Gynecol Oncol, 63, 247-53.

Gadducci A, Cosio S, Romanini A, Genazzani AR (2008). The management of patients with uterine sarcoma: A debated clinical challenge. Crit Rev Oncol Hematol, 65, 129-42.

Gadduci A (2011). Prognostic factors in uterine sarcoma. Best Pract Res Clin Obstet Gynaecol, 25, 783-95.

Garg G, Shah JP, Toy EP, et al (2010). Stage IA vs IB endometrial stromal sarcoma: does the new staging system predict survival? Gynecol Oncol, 118, 8-13.

Geller MA, Argenta P, Bradley W, et al (2004). Treatment and recurrence in endometrial stromal sarcomas and the relation to c-kit expression. Gynecol Oncol, 95, 632-6.

Ghaemmaghami F, Zarchi MK, Gilani MM, et al (2008). Uterine sarcoma: Clinicopathological characteristics, treatment and outcome in İran. Asian Pac J Cancer Prev, 9, 421-6.

Goff BA, Rice LW, Fleishhacker D, et al (1993). Uterine leiomyosarcoma and endometrial stromal sarcoma: lymph node metastases and sites of recurrence. Gynecol Oncol, 50, 105-9.

Haberal A, Kayikcioglu F, Boran N, et al (2003). Endometrial stromal sarcoma of the uterus: analysis of 25 patients. Eur J Obstet Gynecol Reprod Biol, 109, 209-13.

Ihnen M, Mahner S, Jänicke F, Schwarz J (2007). Current treatment options in uterine endometrial stromal sarcoma: Report of a case and review of the literature. Int J Gynecol Cancer, 17, 957-63.

Koss LG, Spiro RH, Brunschwig A (1965). Endometrial stromal sarcoma. Surg Gynecol Obstet, 121, 531-7.

Larson B, Silfversward C, Nilsson B, Pettersson F (1990). Endometrial stromal sarcoma of the uterus. A clinical and histopathological study. The Radiumhemmet series 19361981. Eur J Obstet Gynecol Reprod Biol, 35, 239-49.

Leath CA, Huh WK, Hyde J, et al (2007). A multi-institutional review of outcomes of endometrial stromal sarcoma. Gynecol Oncol, 105, 630-4.

Li AJ, Giuntoli RL, Drake R, et al (2005). Ovarian preservation in stage I low-grade endometrial stromal sarcomas. Obstet Gynecol, 106, 1304-8.

Li N, Wu LY, Zhang HT, et al (2008). Treatment options in stage I endometrial stromal sarcoma: a retrospective analysis of 53 cases. Gynecol Oncol, 108, 306-11.

Malouf GG, Duclos J, Rey A, et al (2010). Impact of adjuvant treatment modalities on the management of patients with stages I-II endometrial stromal sarcoma. Ann Oncol, 21, 2102-6.

Maluf FC, Sabatini P, Schwartz L, Xia J, Aghajanian C (2001). Endomerial stromal sarcoma: objective response to letrozole. Gynecol Oncol, 82, 384-8.

Mansi J.L, Ramachandra S, Wiltshaw E, Fisher C (1990). Endometrial stromal sarcomas. Gynecol Oncol, 36, 113-8.

Moinfar F, Azodi M, Tavassoli FA (2007). Uterine sarcomas. Pathology, 39, 55-71.

Nicolas ML, Cathro HP, Kerr SE, Stelow EB (2007). Cytomorphologic features of low-grade endometrial stromal sarcoma. AJR Am J Clin Pathol, 128, 265-71.

Nordal RR, Kristensen GB, Kaern J, et al (1996). The prognostic significance of surgery, tumor size, malignancy grade, menopausal status, and DNA ploidy in endometrial stromal sarcoma. Gynecol Oncol, 62, 254-9.

Norris HJ, Taylor HB (1966). Mesenchymal tumors of the uterus. I. A clinical and pathological study of 53 endometrial stromal tumors. Cancer, 19, 755-66.

Prat J (2009). FIGO staging for uterine sarcomas. Int J Gynaecol Obstet, 104, 177-8.

Rauh-Hain JA, del Carmen MG (2013). Endometrial stromal 

sarcoma: a systematic review. Obstet Gynecol, 122, 676-83.

Reich O, Winter R, Regauer S (2005). Should lymphadenectomy be performed in patients with endometrial stromal sarcoma? Gynecol Oncol, 97, 982-3.

Riopel J, Plante M, Renaud MC, Roy M, Tetu B (2005). Lymph node metastases in low-grade endometrial stromal sarcoma. Gynecol Oncol, 96, 402-6.

Sagage S, Yamashita K, Ishioka S, et al (2004). Preoperative diagnosis and treatment results in 106 patients with uterine sarcoma in Hokkaido, Japan. Oncology, 67, 33-9

Spano JP, Soria JC, Kambouchner M, et al (2003). Long-term survival of patients given hormonal therapy for metastatic endometrial stromal sarcoma. Med Oncol, 20, 87-93.

Tanz R, Mahfoud T, Bazine A, et al (2012). Endometrial stromal sarcoma: prognostic factors and impact of adjuvant therapy in early stages. Hematol Oncol Stem Cell Ther, 5, 31-5.

Tavassoli FA, Devilee P(2003) .World Health Organization classification of tumors. Mesenchymal tumors and related lesions. In: 'Pathology and genetics of tumors of the breast and female organs', Eds.Tavassoli FA and Devilee P. IARC Press, Lyon( France), 233-6.

Weitmann HD, Knocke TM, Kucera H, Potter R (2001). Radiation therapy in the treatment of stromal sarcoma. Int $J$ Radiat Oncol Biol Phys, 41, 739-48.

Xue WC, Cheung AN (2011). Endometrial stromal sarcoma of the uterus. Best Pract Res Clin Obstet Gynaecol, 25, 719-32.

Yamawaki T, Shimizu Y, Hasumi K (1997). Treatment of stage IV "high-grade" endometrial stromal sarcoma with ifosfamide, adriamycin, and cisplatin. Gynecol Oncol, 64, 265-9. 